\title{
A systematic review of the effectiveness of knowledge translation interventions for chronic noncancer pain management
}

\author{
Maria B Ospina BSc MSc PhD ${ }^{1}$, Paul Taenzer PhD², Saifee Rashiq MB MSc FRCPC ${ }^{3}$, \\ Joy C MacDermid BSc BScPT MSc PhD ${ }^{4}$, Eloise Carr BSc (Hons) RN PGCEA RNT MSc PhD ${ }^{5}$, \\ Dagmara Chojecki MLIS ${ }^{1}$, Christa Harstall BSc MLS MHSA${ }^{1}$, James L Henry PhD ${ }^{6}$
}

\begin{abstract}
MB Ospina, P Taenzer, S Rashiq, et al. A systematic review of the effectiveness of knowledge translation interventions for chronic noncancer pain management. Pain Res Manag
\end{abstract} 2013;18(6):e129-e141.

BACKGROUND: Reliable evidence detailing effective treatments and management practices for chronic noncancer pain exists. However, little is known about which knowledge translation (KT) interventions lead to the uptake of this evidence in practice.

OBJECTIVES: To conduct a systematic review of the effectiveness of KT interventions for chronic noncancer pain management.

METHODS: Comprehensive searches of electronic databases, the gray literature and manual searches of journals were undertaken. Randomized controlled trials, controlled clinical trials and controlled before-and-after studies of KT interventions were included. Data regarding interventions and primary outcomes were categorized using a standard taxonomy; a riskof-bias approach was adopted for study quality. A narrative synthesis of study results was conducted.

RESULTS: More than 8500 titles and abstracts were screened, with 230 full-text articles reviewed for eligibility. Nineteen studies were included, of which only a small proportion were judged to be at low risk of bias. Interactive KT education for health care providers has a positive effect on patients' function, but its benefits for other health provider- and patient-related outcomes are inconsistent. Interactive education for patients leads to improvements in knowledge and function. Little research evidence supports the effectiveness of structural changes in health systems and quality improvement processes or coordination of care.

CONCLUSIONS: KT interventions incorporating interactive education in chronic noncancer pain led to positive effects on patients' function and knowledge about pain. Future studies should provide implementation details and use consistent theoretical frameworks to better estimate the effectiveness of such interventions.

Key Words: Chronic pain; Education; Knowledge translation; Systematic review

Chronic noncancer pain is a poorly understood condition in which the patient experiences pain for prolonged periods of time, often despite the healing or complete absence of demonstrable tissue damage. More complex in nature than acute pain, chronic noncancer pain is often intertwined with multiple physical and psychosocial complications, and accompanied by other medical disorders that further increase the burden of this disease. Issues such as immobility, wasting of muscle and joints, depression of the immune system, poor appetite and nutrition, dependence on medication, overuse and/or inappropriate use of the health care system, poor job performance or inability to work, and anxiety have all been reported in the chronic pain literature, along with chronic comorbidities such

\section{Une analyse systématique de l'efficacité des interventions de transfert du savoir dans la prise en charge de douleurs non cancéreuses chroniques}

HISTORIQUE : Il existe des données fiables exposant des traitements et des pratiques de prise en charge efficaces de la douleur non cancéreuse chronique. Cependant, on ne sait pas vraiment quelles interventions de transfert du savoir (TS) favorisent l'exécution de cet aspect de la pratique. OBJECTIFS : Procéder à une analyse systématique de l'efficacité des interventions de TS pour la prise en charge de la douleur non cancéreuse chronique.

MÉTHODOLOGIE : Les chercheurs ont effectué des recherches approfondies dans des bases de données électroniques, des documents non publiés et des revues papier. Ils ont inclus des essais aléatoires et contrôlés, des essais cliniques contrôlés et des études contrôlées avant-après des interventions de TS. Ils ont classé les données sur les interventions et les résultats primaires à l'aide d'une taxonomie standard. Ils ont adopté une approche de risque de biais pour la qualité de l'étude et procédé à une synthèse narrative des résultats de l'étude.

RÉSULTATS : Les chercheurs ont fouillé plus de 8500 articles et résumés et 230 articles intégraux en vue d'évaluer leur admissibilité. Dix-neuf études ont été incluses dans l'étude, dont seulement une petite proportion était considérée comme à faible risque de biais. L'enseignement interactif du TS à l'intention des dispensateurs de soins a un effet positif sur la fonction des patients, mais ses avantages sur les résultats liés aux autres dispensateurs de soins et aux patients ne sont pas uniformes. L'éducation interactive des patients s'associe à des améliorations du savoir et de la fonction. Peu de données de recherches appuient l'efficacité des changements structurels aux systèmes de santé et aux processus d'amélioration de la qualité ou de coordination des soins.

CONCLUSIONS : Les interventions de TS qui intègrent l'éducation interactive en matière de douleur non cancéreuse chronique ont des effets positifs sur la fonction des patients et les connaissances sur la douleur. De prochaines études devraient contenir de l'information sur la mise en œuvre et faire appel à des cadres théoriques pour mieux évaluer l'efficacité de ces interventions.

as heart disease, depression and sleep disorders (1-4). As a result of these issues, studies have shown that the health-related quality of life of chronic noncancer pain patients is among the lowest observed for any medical condition (5).

Chronic noncancer pain in Canada, as well as in many other parts of the world, is a widespread and burdensome problem for individuals, the health care system, the economy and society as a whole (1). Some studies indicate that large numbers of Canadians (upwards of 20\% [6] to 30\% [7]) report some form of chronic persistent pain, lasting an average of 10.7 years. Chronic noncancer pain results in significant suffering, disability and reduced quality of life (8), and significant personal (9) and societal economic burdens, estimated to exceed $\$ 6$ billion annually (10).

${ }^{1}$ Institute of Health Economics, Edmonton; ${ }^{2}$ Departments of Psychiatry, Medicine and Oncology, Faculty of Medicine, University of Calgary, Calgary;

${ }^{3}$ Department of Anesthesiology and Pain Medicine, University of Alberta, Edmonton, Alberta; ${ }^{4}$ School of Rehabilitation Sciences, McMaster

University, Hamilton, Ontario; ${ }^{5}$ Faculty of Nursing, University of Calgary, Calgary, Alberta; ${ }^{6}$ McMaster University, Hamilton, Ontario

Correspondence: Maria B Ospina, Suite 1200, 10405 Jasper Avenue Northwest, Edmonton, Alberta T5J 3N4.

Telephone 780-448-4881, fax 780-448-0018, e-mail mospina@ihe.ca 
Not surprisingly, it has been further identified that chronic noncancer pain places a significant strain on the health care system. For instance, it has been found that these individuals make more physician and emergency room visits and experience longer hospital stays than those without such disorders $(11-14)$. High resource use by chronic noncancer pain patients leads to high direct costs for care $(13,15,16)$ in addition to high indirect costs related to significant decreases in work productivity and disability payments $(14,17-20)$. The scientific literature overwhelmingly points to the significant burden that chronic noncancer pain, a disease and health care problem in its own right, places on the individual, the health care system and society as a whole.

Despite the fact that reliable evidence exists (eg, guidelines, systematic reviews, etc) detailing effective treatments and management practices for chronic noncancer pain, evidence-based treatments and practices are often not used by practitioners, leading to these conditions being undertreated and mismanaged $(1,21-24)$.

The answer advocated by many to this dilemma lies in the area of knowledge translation (KT) because the problem encompasses the gap between the knowledge that exists about managing chronic noncancer pain and current health care practices (1). KT has been defined in Canada as:

The exchange, synthesis and ethically-sound application of knowledge - within a complex system of interactions among researchers and users - to accelerate the capture of the benefits of research for Canadians through improved health, more effective services and products, and a strengthened health care system (25).

$\mathrm{KT}$ is based on the notion that the quality of care and the health of the population will increase when relevant research findings and evidence are applied to everyday practice $(26,27)$. KT, however, is not a simple, straightforward process. Multiple factors, such as the everincreasing volume of literature, lack of resources, and other individual and organizational constraints, often complicate and impede attempts to incorporate evidence into practice (26,28-30). KT research attempts to identify effective interventions that address these barriers and lead to evidence uptake and improvement in patient outcomes (30).

Little is known about which KT interventions are specifically effective in the area of chronic noncancer pain management (1). The aim of the present systematic review was to systematically locate and assess the evidence regarding the effectiveness of KT interventions for chronic noncancer pain management.

\section{Identification of studies}

The protocol of the present study was registered in the PROSPERO international prospective register of systematic reviews (2011:CRD42011001379). The methodology followed the standards recommended by the Cochrane Effective Practice and Organisation of Care Review Group (EPOC) guidelines for conducting systematic reviews of interventions designed to improve the delivery, practice and organization of health care services (31). Comprehensive searches of psychological, sociological and biomedical electronic databases (The Cochrane Library, MEDLINE, EMBASE, Centre for Reviews and Dissemination Database, Cumulative Index to Nursing and Allied Health, Web of Science, PsycINFO, Sociological Abstracts, SocINDEX, Social Services Abstracts, ABI Inform, Business Source Complete, Health-evidence.ca, KT+, Campbell Collaboration, Knowledge Utilization - Utilisation des Connaissances, Canadian Research Index) were conducted from database inception date to September 2011 with no date limits applied. The search strategy was designed by an information specialist and comprised both selected subject headings and keywords relating to chronic pain conditions and KT (the full search strategy is available upon request). In addition, the Cochrane EPOC Register was searched for studies not identified in previous electronic searches. Reference lists of reviews and retrieved articles were checked for relevant studies. Scientific meeting proceedings, clinical trial registries, government documents, theses and dissertations were sought to identify unpublished studies. Language restrictions were not imposed in the searches.

\section{Inclusion and exclusion criteria}

Randomized controlled trials (RCTs), controlled clinical trials (CCTs) and controlled before-and-after studies (CBAs) of KT interventions targeting health care providers working with chronic noncancer pain patients, or individuals with chronic noncancer pain were included. Both individual and cluster designs were considered. A KT intervention was defined as the act of transmitting specific evidence-based knowledge about chronic noncancer pain. To include any given study, it was necessary to be able to identify the source and content of the information transmitted, and to confirm that the information was indeed based on evidence. For example, an experiment to test the effectiveness of informing providers or patients about what the investigators regarded to be best practice in a particular pain condition would not have been included unless it could be verified that the content of the program was entirely derived from scientific evidence and not from opinion or conjecture. An experiment to test the effectiveness of an evidence-based treatment guideline, on the other hand, would have been eligible for inclusion. This stance was taken to restrict the focus of the present study to true KT interventions and, thus, to distinguish it from reviews of the effectiveness of patient and provider education in general, in the belief that KT studies, while less plentiful, deliver a higher quality of factual information.

Outcomes of interest were primary outcomes related to patients (eg, health status, pain intensity and relief, knowledge, satisfaction with care, adverse events, work-related measures), to health providers (eg, changes in practice, knowledge, satisfaction) and to the organization or system (eg, staff turnover, patient workload). Studies were not excluded on the basis of language of publication. Two pairs of reviewers independently screened the titles and abstracts generated from the search strategies to identify potentially relevant articles. The full text of articles deemed relevant and those whose abstracts and titles provided insufficient information were retrieved for a closer inspection by two independent reviewers who determined study eligibility for the review. Disagreements about inclusion and exclusion of studies were resolved through discussions among reviewers until consensus was reached.

\section{Risk of bias assessment}

Two reviewers independently assessed the risk of bias of individual studies using the criteria developed by the Cochrane EPOC group (31). Briefly, RCTs, CCTs and CBAs were assessed for adequacy with regard to 10 validity criteria: sequence generation; allocation concealment; baseline outcome measurement; baseline characteristics; blinding of participants and personnel; blinding or objective assessment of the primary outcome; completeness of follow-up; completeness of outcome reporting; protection against contamination; and other potential threats to validity. Discrepancies in risk of bias assessment between reviewers were resolved by consensus. Risk of bias summaries were reported in relation to the primary outcome reported in the individual studies (32). Studies were not excluded from the analysis because of poor methodological quality.

\section{Data extraction and analysis}

Details regarding study characteristics (ie, design, country, clinical topic, units of allocation and analysis), type of KT intervention, sample size, primary study outcomes and associated statistical significance were extracted from individual studies using a pretested data extraction form and summarized in evidence tables. Studies were grouped according to target audience (ie, health professionals, patients, miscellaneous groups) and then classified according to the Cochrane EPOC taxonomy of interventions (31) into: professional (eg, distribution of educational material, educational meetings and outreach visits, use of local opinion leaders, and audit and feedback), organizational (eg, revision of professional roles, communication systems or equipment availability), financial (eg, various methods of remuneration or payment systems), regulatory interventions (eg, any health service change introduced by regulation or law) and those related to patients and consumer communication (eg, information, health promotion, skills training, coordination of care, supportive environments). 
Details about implementation of the interventions (ie, delivery format, manual availability and training of providers) were obtained. The level of action to support research implementation into practice was classified according to a taxonomy that focuses on the primary goal of KT (33) as: increase awareness of the problem or knowledge gap; acquire new knowledge; evaluate/synthesize new knowledge; make a decision; adaptation of evidence to context; implement/operationalize specific actions (implementation fidelity); facilitate the process of change; and quality/outcome evaluation/monitoring.

Substantial diversity in study designs, methodological quality, KT interventions and outcomes across studies precluded combining individual results into a meta-analysis. Instead, a narrative synthesis of effectiveness outcomes across KT interventions was undertaken according to the guidelines suggested by Popay et al (34). Subgroups were created to guide the exploration of outcomes within classes of KT interventions in the narrative synthesis. The focus of the analysis was the effect of KT interventions on primary outcomes (ie, clearly defined as such by the authors of individual studies or those first listed in the results section). Outcome reporting was divided into patient-related, professional and system outcomes. The timeframes for outcome assessment were grouped into short- (ie, $<3$ months), medium- (ie, between three and six months) and long-term (ie, $>6$ months) outcomes. The initial phases of the review (study screening and eligibility) were managed using the Early Review Organizing Software $(35,36)$; risk of bias assessment, data extraction and narrative synthesis were managed using Excel (Microsoft Corporation, USA).

\section{RESULTS}

\section{Search results}

The search strategy (including electronic and manual sources) identified 13,387 citations. After screening 8765 titles and abstracts, 233 articles were judged to be potentially relevant, 28 of which satisfied the eligibility criteria. Of these, nine references were multiple publications and, therefore, the review included 19 unique studies (seven RCTs [22,37-42], six cluster RCTs [43-48], three CCTs [49-51] and three CBAs [52-54]) reported in 28 publications (Figure 1). The complete list of excluded studies and reasons for exclusion is available on request.

\section{General characteristics of studies}

The studies were mainly conducted in the Netherlands $(40,44,47,51)$ and the United States $(39,42,49,54)$, followed by Canada $(22,46)$, France (37,38), Germany (41,43), Australia (52), Mexico (50), Scotland (53), Spain (45) and the United Kingdom (48). The unit of allocation was the health provider in the majority of studies $(38-40,47,49-51)$, followed by patients $(22,37,41,42)$, professional practices $(43,44,53,54)$ or geographic areas $(45,46,52)$. The clinical topic of KT interventions in the studies included low back pain $(22,37,40,41,43,44,48,49,51-53)$, migraine $(38,42,47)$, osteoarthritis $(46)$ and nonspecific chronic pain conditions $(39,45,50,54)$.

\section{Types of KT interventions}

Thirteen studies $(38-40,43-49,51,53,54)$ examined KT interventions targeting health professionals solely, whereas four studies $(22,37,41,42)$ targeted chronic noncancer pain patients. Two studies assessed KT interventions offered to combined target groups (ie, community [52], and both health professionals and patients [50]). Characteristics and components of KT interventions assessed in individual studies are described in Table 1. Professional educational interventions targeting health providers were the predominant $\mathrm{KT}$ intervention evaluated $(38-40,43-49,51,54)$. The interventions consisted of various educational approaches (eg, distribution of educational materials, educational meetings, educational outreach visits, behavioural instructions and advice in relation to pain management by means of verbal, written, audio- or videotaped or computer-aided modalities) and several additional strategies including reminders $(44,45)$, audit and feedback techniques (49) and local opinion leaders (48). Professional interventions for health providers involving structural changes in the organization and delivery

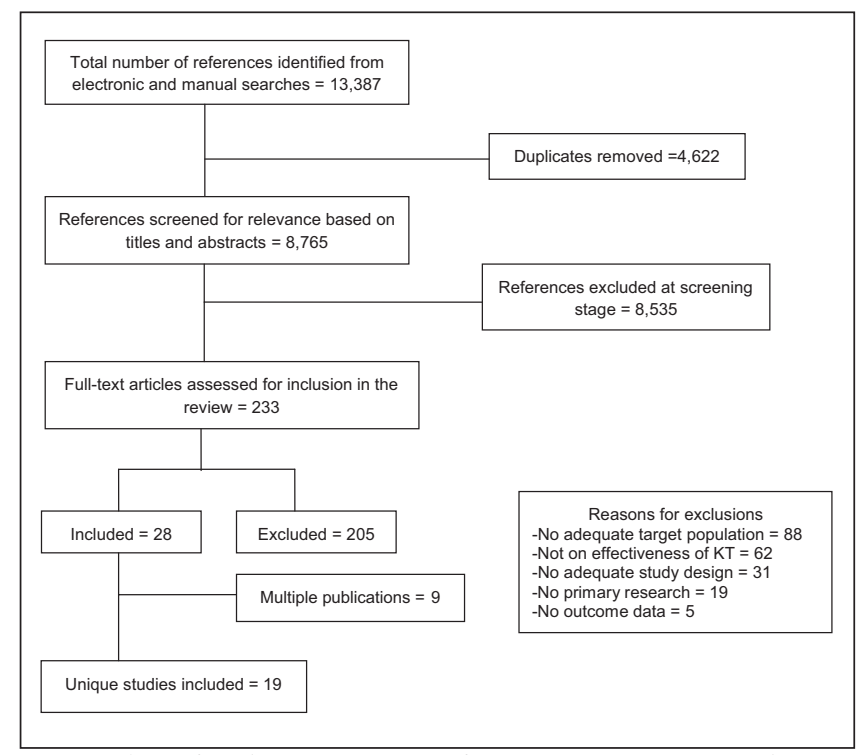

Figure 1) Preferred Reporting Items for Systematic Reviews and MetaAnalyses (PRISMA) flow diagram. KT Knowledge translation

of care (ie, quality improvement) were assessed in one study (53). Health professionals targeted by KT interventions in the studies were mainly general practitioners $(38-40,43,45-47,52)$ followed by physical therapists $(40,44,48,53)$, family physicians and general practitioners $(39,45,50)$, occupational physicians $(49,51)$ and nurses $(54)$.

Interventions targeting chronic noncancer pain patients used information/education and decision aids $(37,41,42)$, or implemented coordination-of-care approaches (22). The studies that assessed KT interventions for various target groups combined professional interventions (ie, educational approaches [50] and mass media [52]) and consumer communication strategies (ie, health promotion and information [52] and education [50]) around chronic noncancer pain issues.

Implementation of KT interventions: The majority of KT interventions were delivered through formats that combined both oral communication and written materials $(41,43-47,49-51,54)$. Other delivery formats included Internet-based applications $(38,39,42,53)$, videos $(39,50,52,54)$ and interactive computer programs $(39,42)$. Health professionals mainly participated in the delivery of KT interventions $(22,41,43-46,48-50,54)$; details of the qualifications of intervention providers, however, were not described uniformly across the studies. Overall, the studies failed to consistently report important details of the implementation of KT interventions such as the theoretical framework of behaviour change, the frequency and duration of delivery, training of providers and evaluation of intervention fidelity. The KT interventions identified in the present review supported the implementation of research into practice in many ways. The majority of them promoted the acquisition of new knowledge about chronic noncancer pain issues $(37-51,54)$, or contributed to the implementation of clinical practice guidelines in clinical decision-making (22,38,42-44,46,47,50-54). Some KT interventions were aimed at increasing awareness of chronic noncancer pain problems and the knowledge gaps in the field $(37,41,43,48-50,52)$. Other KT interventions were aimed at facilitating processes of behaviour change and monitoring quality of care $(44,45,49,53,54)$. Less frequently, KT interventions in chronic noncancer pain were aimed at evaluating or synthesizing new knowledge $(42,46)$ or adapting evidence to context $(22,53)$.

\section{Risk of bias}

The risk of bias in individual studies is reported in Table 2. The high risk of bias components across the studies included inadequate (or lack of) random sequence generation $(37,48-54)$ and allocation concealment (49-54), differences at baseline measurement $(22,43,45,46,52,54)$ and inadequate account for losses to follow-up $(38-40,45,51,54)$. 


\section{TABLE 1}

Knowledge translation intervention types evaluated in individual studies

\begin{tabular}{|c|c|c|c|c|c|c|c|c|c|c|}
\hline \multirow{2}{*}{$\begin{array}{l}\text { Author } \\
\text { (reference), } \\
\text { year }\end{array}$} & \multirow[b]{2}{*}{ EPOC classification } & \multirow{2}{*}{\multicolumn{2}{|c|}{ Intervention characteristics and target group }} & \multicolumn{7}{|c|}{$\begin{array}{c}\text { Level of action to support } \\
\text { moving evidence into practice }\end{array}$} \\
\hline & & & & 1 & 2 & 3 & 4 & 5 & 6 & 7 \\
\hline \multicolumn{11}{|c|}{ Health professionals } \\
\hline $\begin{array}{l}\text { Becker et } \\
\text { al (43), } \\
2008\end{array}$ & $\begin{array}{l}\text { Professional (distribution of } \\
\text { educational materials; } \\
\text { educational meetings; } \\
\text { educational outreach visit) }\end{array}$ & $\begin{array}{l}\text { Clinical practice guideline implementation (GPs) } \\
\text { Format: Oral communication, written material } \\
\text { Delivered by: Health professional } \\
\text { Frequency: } 4 \text { sessions }\end{array}$ & $\begin{array}{l}\text { Duration: NR } \\
\text { Theoretical framework: NR } \\
\text { Manual availability: No } \\
\text { Training of providers: NR }\end{array}$ & Yes & Yes 1 & & Yes & No & No & No \\
\hline $\begin{array}{l}\text { Bekkering } \\
\text { et al } \\
(44) \\
2005\end{array}$ & $\begin{array}{l}\text { Professional (distribution of } \\
\text { educational materials; } \\
\text { educational meetings; } \\
\text { reminders) }\end{array}$ & $\begin{array}{l}\text { Clinical practice guideline dissemination (physical therapists) } \\
\text { Format: Oral communication, written material, role-playing } \\
\text { with actors, reminders } \\
\text { Delivered by: Health professional, researcher, self- } \\
\text { administered } \\
\text { Frequency: } 2 \text { sessions }\end{array}$ & $\begin{array}{l}\text { Duration: } 2.5 \mathrm{~h} \\
\text { Theoretical framework: } \\
\text { Changing behaviour } \\
\text { model } \\
\text { Manual availability: No } \\
\text { Training of providers: No }\end{array}$ & No & Yes 1 & No & Yes & No & No & Yes \\
\hline $\begin{array}{l}\text { Derebery } \\
\text { et al } \\
(49) \\
2002\end{array}$ & $\begin{array}{l}\text { Professional (distribution of } \\
\text { educational materials; } \\
\text { educational meetings; } \\
\text { audit and feedback) }\end{array}$ & $\begin{array}{l}\text { Active evidence-based educational package (occupational } \\
\text { physicians) } \\
\text { Format: Oral communication, written material } \\
\text { Delivered by: Health professional, self-administered } \\
\text { Frequency: } 1 \text { session }\end{array}$ & $\begin{array}{l}\text { Duration: } 2 \mathrm{~h} \\
\text { Theoretical framework: } \\
\text { Bio-psychosocial model } \\
\text { of management } \\
\text { Manual availability: NR } \\
\text { Training of providers: NR }\end{array}$ & Yes & Yes 1 & No & No & No & No & Yes \\
\hline $\begin{array}{l}\text { Figueiras } \\
\text { et al } \\
(45) \\
2001\end{array}$ & $\begin{array}{l}\text { Professional (distribution of } \\
\text { educational materials; } \\
\text { educational outreach visit; } \\
\text { reminders) }\end{array}$ & $\begin{array}{l}\text { One-to-one education (GPs, FPs) } \\
\text { Format: Oral communication, written material } \\
\text { Delivered by: Health professional } \\
\text { Frequency: } 1 \text { session }\end{array}$ & $\begin{array}{l}\text { Duration: } 20 \text { min } \\
\text { Theoretical framework: NR } \\
\text { Manual availability: No } \\
\text { Training of providers: Yes }\end{array}$ & No & Yes 1 & No & No & No & No & Yes \\
\hline $\begin{array}{l}\text { Geraud et } \\
\text { al (28), } \\
2009\end{array}$ & $\begin{array}{l}\text { Professional (distribution of } \\
\text { educational materials) }\end{array}$ & $\begin{array}{l}\text { E-learning education based on clinical practice guideline } \\
\text { (GPs) } \\
\text { Format: Internet-based } \\
\text { Delivered by: Computer/multimedia } \\
\text { Frequency: NR }\end{array}$ & $\begin{array}{l}\text { Duration: NR } \\
\text { Theoretical framework: NR } \\
\text { Manual availability: No } \\
\text { Training of providers: No }\end{array}$ & No & Yes 1 & No & Yes & No & No & No \\
\hline $\begin{array}{l}\text { Harris et al } \\
(39) \\
2008\end{array}$ & $\begin{array}{l}\text { Professional (distribution of } \\
\text { educational materials) }\end{array}$ & $\begin{array}{l}\text { Evidence-based online CME pain program (GPs, FPs) } \\
\text { Format: Videos, interactive computer programs, Internet-based } \\
\text { Delivered by: Researcher, computer/multimedia, self- } \\
\text { administered } \\
\text { Frequency: } 1 \text { session }\end{array}$ & $\begin{array}{l}\text { Duration: } 4 \mathrm{~h} \\
\text { Theoretical framework: NR } \\
\text { Manual availability: NA } \\
\text { Training of providers: NA }\end{array}$ & No & Yes 1 & No & No & No & No & No \\
\hline $\begin{array}{l}\text { Jones et al } \\
(54) \\
2004\end{array}$ & $\begin{array}{l}\text { Professional (distribution of } \\
\text { educational materials; } \\
\text { educational meetings; } \\
\text { educational outreach visit; } \\
\text { opinion leaders) }\end{array}$ & $\begin{array}{l}\text { Interactive educational program (nurses) } \\
\text { Format: Oral communication, Written material, videos } \\
\text { Delivered by: Health professional, researcher, computer/ } \\
\text { multimedia, self-administered } \\
\text { Frequency: } 4 \text { sessions }\end{array}$ & $\begin{array}{l}\text { Duration: } \\
\text { Theoretical framework: NR } \\
\text { Manual availability: No } \\
\text { Training of providers: No }\end{array}$ & No & Yes 1 & No & Yes & No & No & Yes \\
\hline $\begin{array}{l}\text { Keijsers et } \\
\text { al (40), } \\
1992\end{array}$ & $\begin{array}{l}\text { Professional (distribution of } \\
\text { educational materials) }\end{array}$ & $\begin{array}{l}\text { Provision of written empirical evidence on the efficacy of } \\
\text { back school treatment (GPs, physical therapists) } \\
\text { Format: Written material } \\
\text { Delivered by: Self-administered } \\
\text { Frequency: NR }\end{array}$ & $\begin{array}{l}\text { Duration: NR } \\
\text { Theoretical framework: NR } \\
\text { Manual availability: NA } \\
\text { Training of providers: NA }\end{array}$ & No & Yes 1 & No & No & No & No & No \\
\hline $\begin{array}{l}\text { Rahme et } \\
\text { al (46), } \\
2005\end{array}$ & $\begin{array}{l}\text { Professional (distribution of } \\
\text { educational materials; } \\
\text { educational meetings) }\end{array}$ & $\begin{array}{l}\text { Workshop and decision tree (GPs) } \\
\text { Format: Oral communication, written material } \\
\text { Delivered by: Health professional, decision aids } \\
\text { Frequency: } 1 \text { session }\end{array}$ & $\begin{array}{l}\text { Duration: } 90 \text { min } \\
\text { Theoretical framework: NR } \\
\text { Manual availability: NA } \\
\text { Training of providers: NA }\end{array}$ & No & Yes & Yes & Yes & No & No & No \\
\hline $\begin{array}{l}\text { Smelt et al } \\
(47) \text {, } \\
2010\end{array}$ & $\begin{array}{l}\text { Professional (distribution of } \\
\text { educational materials; } \\
\text { educational meetings) }\end{array}$ & $\begin{array}{l}\text { Proactive education intervention (GPs) } \\
\text { Format: Oral communication, written material } \\
\text { Delivered by: NR } \\
\text { Frequency: NR }\end{array}$ & $\begin{array}{l}\text { Duration: NR } \\
\text { Theoretical framework: NR } \\
\text { Manual availability: No } \\
\text { Training of providers: No }\end{array}$ & & Yes 1 & No & Yes & No & No & No \\
\hline $\begin{array}{l}\text { Smits et al } \\
(51) \\
2000\end{array}$ & $\begin{array}{l}\text { Professional (distribution of } \\
\text { educational materials; } \\
\text { educational meetings) }\end{array}$ & $\begin{array}{l}\text { Posgraduate educational program based on the Deutch } \\
\text { Clinical practice guideline for pain rehabilitation } \\
\text { (occupational physicians) } \\
\text { Format: Oral communication, written material } \\
\text { Delivered by: NR } \\
\text { Frequency: } 7.5 \text { days }\end{array}$ & $\begin{array}{l}\text { Duration: NR } \\
\text { Theoretical framework: } \\
\text { Miller's pyramid of clinical } \\
\text { assessment } \\
\text { Manual availability: NR } \\
\text { Training of providers: NR }\end{array}$ & No & Yes 1 & No & Yes & No & No & No \\
\hline
\end{tabular}


TABLE 1 - CONTINUED

Knowledge translation intervention types evaluated in individual studies

\begin{tabular}{|c|c|c|c|c|c|c|c|c|c|c|}
\hline \multirow{2}{*}{$\begin{array}{l}\text { Author } \\
\text { (reference), } \\
\text { year }\end{array}$} & \multirow{2}{*}{ EPOC classification } & \multirow{2}{*}{\multicolumn{2}{|c|}{ Intervention characteristics and target group }} & \multicolumn{7}{|c|}{$\begin{array}{c}\text { Level of action to support } \\
\text { moving evidence into practice }\end{array}$} \\
\hline & & & & 1 & 2 & 3 & 4 & 5 & 6 & 7 \\
\hline \multicolumn{11}{|c|}{ Health professionals - Continued } \\
\hline $\begin{array}{l}\text { Stevenson } \\
\text { et al } \\
(48) \\
2006\end{array}$ & $\begin{array}{l}\text { Professional (distribution of } \\
\text { educational materials; } \\
\text { local opinion leaders; } \\
\text { educational outreach } \\
\text { visits) }\end{array}$ & $\begin{array}{l}\text { Evidence-based educational package (physical therapists) } \\
\text { Format: Oral communication } \\
\text { Delivered by: Health professional } \\
\text { Frequency: } 2 \text { sessions }\end{array}$ & $\begin{array}{l}\text { Duration: } 2.5 \mathrm{~h} \\
\text { Theoretical framework: } \\
\text { Opinion leaders' theory } \\
\text { Manual availability: No } \\
\text { Training of providers: No }\end{array}$ & Yes & Yes & No & No $N$ & No & No & No \\
\hline $\begin{array}{l}\text { Ferguson } \\
\text { et al } \\
(53) \\
2010\end{array}$ & $\begin{array}{l}\text { Structural (quality } \\
\text { improvement; audit and } \\
\text { feedback) }\end{array}$ & $\begin{array}{l}\text { Quality improvement and audit (physical therapists) } \\
\text { Format: Internet-based } \\
\text { Delivered by: Computer/multimedia, self-administered } \\
\text { Frequency: NR }\end{array}$ & $\begin{array}{l}\text { Duration: NR } \\
\text { Theoretical framework: } \\
\text { Embedded research model } \\
\text { Manual availability: No } \\
\text { Training of providers: Yes }\end{array}$ & No & No & No & Yes Y & Yes & No & Yes \\
\hline \multicolumn{11}{|l|}{ Patients } \\
\hline $\begin{array}{l}\text { Coudeyre } \\
\text { et al } \\
(37) \\
2006\end{array}$ & $\begin{array}{l}\text { Patients, consumer and } \\
\text { communication } \\
\text { (distribution of educational } \\
\text { materials; information, } \\
\text { consumer decision aids) }\end{array}$ & $\begin{array}{l}\text { The Back Book } \\
\text { Format: Written material } \\
\text { Delivered by: Self-administered } \\
\text { Frequency: NR }\end{array}$ & $\begin{array}{l}\text { Duration: } 3 \text { months } \\
\text { Theoretical framework: NR } \\
\text { Manual availability: NA } \\
\text { Training of providers: NA }\end{array}$ & Yes & Yes & No & No 1 & No & No & No \\
\hline $\begin{array}{l}\text { Meng et al } \\
(41) \text {, } \\
2011\end{array}$ & $\begin{array}{l}\text { Patients, consumer and } \\
\text { communication } \\
\text { (distribution of educational } \\
\text { materials; educational } \\
\text { meetings; information, } \\
\text { consumer decision aids) }\end{array}$ & $\begin{array}{l}\text { Back school progam based on evidence and practice } \\
\text { guidelines (patients) } \\
\text { Format: Oral communication, written material } \\
\text { Delivered by: Health professional } \\
\text { Frequency: } 7 \text { sessions }\end{array}$ & $\begin{array}{l}\text { Duration: } 55 \text { min } \\
\text { Theoretical framework: } \\
\text { Health action process } \\
\text { approach, fear-avoidance } \\
\text { model } \\
\text { Manual availability: Manual } \\
\text { available } \\
\text { Training of providers: Yes }\end{array}$ & Yes & Yes & No & No 1 & No & No & No \\
\hline $\begin{array}{l}\text { Sciamanna } \\
\text { et al (42), } \\
2006\end{array}$ & $\begin{array}{l}\text { Patients, consumer and } \\
\text { communication } \\
\text { (distribution of educational } \\
\text { materials; information, } \\
\text { consumer decision aids) }\end{array}$ & $\begin{array}{l}\text { Internet-based computer program } \\
\text { Format: Internet-based interactive computer programs } \\
\text { Delivered by: Computer/multimedia }\end{array}$ & $\begin{array}{l}\text { Frequency: NR } \\
\text { Duration: NR } \\
\text { Theoretical framework: } \\
\text { Chronic care model } \\
\text { Manual availability: NA } \\
\text { Training of providers: NA }\end{array}$ & No & Yes & Yes & Yes 1 & No & No & No \\
\hline $\begin{array}{l}\text { Rossignol } \\
\text { et al } \\
(22) \\
2000\end{array}$ & $\begin{array}{l}\text { Patients, consumer and } \\
\text { communication } \\
\text { (coordination of care) }\end{array}$ & $\begin{array}{l}\text { Coordination of primary care program } \\
\text { Format: Coordinated care } \\
\text { Delivered by: Health professional } \\
\text { Frequency: NR }\end{array}$ & $\begin{array}{l}\text { Duration: NR } \\
\text { Theoretical framework: NR } \\
\text { Manual availability: No } \\
\text { Training of providers: Yes }\end{array}$ & No & No & No & Yes Y & Yes & No & No \\
\hline \multicolumn{11}{|c|}{ Combined target groups } \\
\hline $\begin{array}{l}\text { Doubova } \\
\text { et al } \\
(50) \\
2010\end{array}$ & $\begin{array}{l}\text { Professional (distribution of } \\
\text { educational materials; } \\
\text { educational meetings; } \\
\text { educational outreach visit) } \\
\text { Patients, consumer and } \\
\text { communication } \\
\text { (distribution of educational } \\
\text { materials; information, } \\
\text { consumer decision aids) }\end{array}$ & $\begin{array}{l}\text { Interactive educational programme (patients and FPs) } \\
\text { Format: Oral communication, written material, videos } \\
\text { Delivered by: Health professional, computer/multimedia } \\
\text { Frequency: } 6 \text { sessions }\end{array}$ & $\begin{array}{l}\text { Duration: } 1 \mathrm{~h} \\
\text { Theoretical framework: NR } \\
\text { Manual availability: NR } \\
\text { Training of providers: No }\end{array}$ & Yes & Yes & No & Yes 1 & No & No & No \\
\hline $\begin{array}{l}\text { Buchbinder } \\
\text { et al (52), } \\
2005\end{array}$ & $\begin{array}{l}\text { Professional (mass media) } \\
\text { Patients, consumer and } \\
\text { communication (health } \\
\text { promotion, information) }\end{array}$ & $\begin{array}{l}\text { Victorian Work Cover Authority back campaign (community) } \\
\text { Format: Written material, videos, televised advertisements } \\
\text { Delivered by: Computer/multimedia } \\
\text { Frequency: NR }\end{array}$ & $\begin{array}{l}\text { Duration: } 1 \text { year } \\
\text { Theoretical framework: NR } \\
\text { Manual availability: NA } \\
\text { Training of providers: NA }\end{array}$ & Yes & No & No & Yes 1 & No & No & No \\
\hline
\end{tabular}

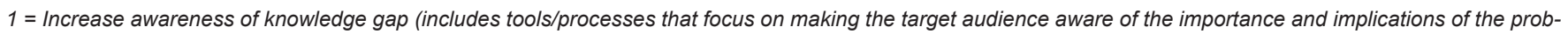

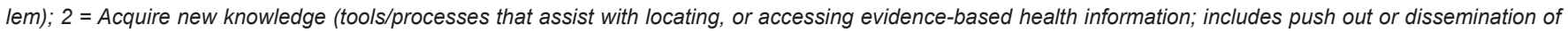

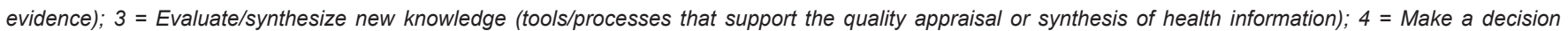

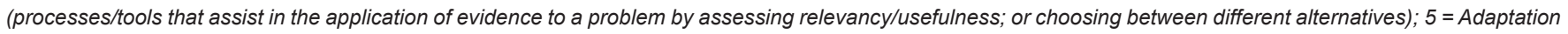

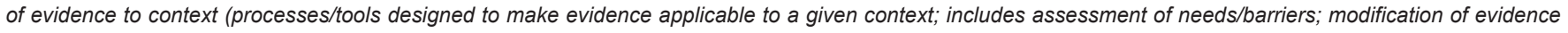

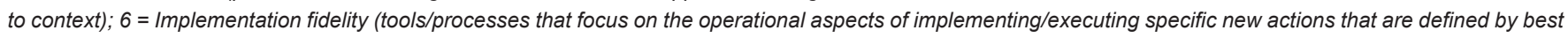

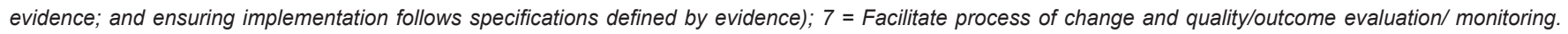

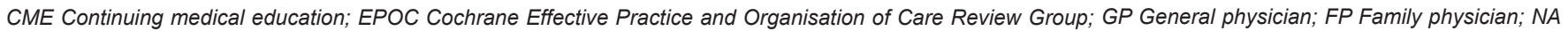
Not applicable; NR Not reported 
TABLE 2

Summary assessment of risk of bias for each study in the review

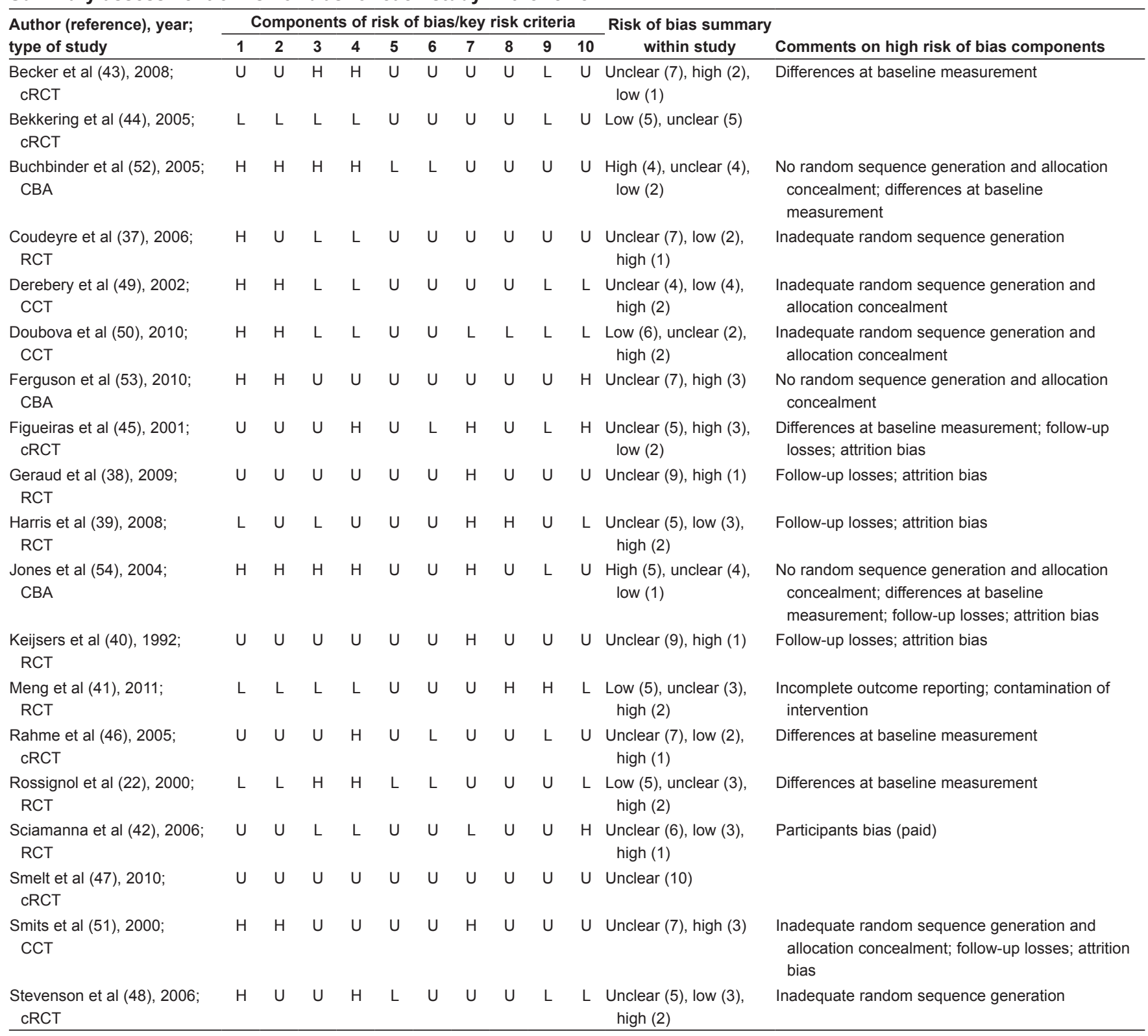

Components of risk of bias criteria: 1 = Sequence generation; 2 = Allocation concealment; 3 = Baseline outcome measurement; 4 = Baseline characteristics; 5 = Participants and personnel blinding; 6 = Blinding or objective assessment of primary outcome; 7 = Completeness of follow-up; $8=$ Complete outcome reporting; $9=$ Protection against contamination; and $10=$ Other potential threats to validity. CBA Controlled before-and-after study; CCT Controlled clinical trial; cRCT Cluster randomized controlled trial; $H$ High risk of bias; L Low risk of bias; RCT Randomized controlled trial; $U$ Unclear risk of bias

Due to poor quality of reporting in some individual studies, the effects of bias related to the lack of blinding of participants and outcome assessment, and selective outcome reporting were unclear. One study was judged to have incomplete outcome reporting (41). Another study was likely to be affected by threats to validity derived from participants' bias (42). The results of the risk of bias assessment presented in Table 2 suggest that a small proportion of studies $(22,41,44)$ were likely to be at low risk of bias.

Effects of KT interventions

More than one-half of the studies $(38-40,45,46,48,50,51,53,54)$ assessed primary outcomes related to the health providers. They included short-term assessments of change in knowledge about pain and pain management $(39,51,54)$, and appropriateness or improvement in prescription behaviour (assessed at medium- [46,50] and long-term [45]). Other health provider outcomes included medium- $(48,53)$ and shortterm (38) changes in clinical management (such as time spent providing advice to patients, or case notes documenting chronic noncancer pain) and assessments of the level of confidence regarding certain therapeutic options (evaluated at short-term [40]). Patient-related outcomes were assessed in nine studies $(22,37,41-44,47,49,52)$. They included short-, medium- and long-term evaluations of patients' function $(43,44)$, medium- and long-term assessments of knowledge acquisition (41) and beliefs about pain (52), long-(49) and medium-term (22) work-related 
outcomes such as restrictions in work duty rate and return to work, and medium-term assessments of pain complaints (47) or discussion of painrelated topics during the clinical encounter (42). Primary outcomes addressing changes at the organization or system level were not identified. Table 3 summarizes the characteristics of intervention groups and $\mathrm{KT}$ effectiveness outcomes according to the target population in the individual studies. The main results of each study are reported in natural units extracted from the studies. The identification of a small number of studies reporting comparable outcome measures precluded the visual assessment of potential publication bias using funnel plots.

KT interventions targeting health professionals: 12 studies assessed the effect of KT professional education-mediated interventions on health provider outcomes (ie, changes in knowledge about pain and pain management $[39,51,54]$, clinical management $[38,48]$, prescription behaviour [45,46], confidence in therapeutic options [40]) and patient-related outcomes (ie, function [43,44], pain complaints [47] and changes in work duty rate [49]).

Professional education-mediated interventions, such as electronic learning education based on clinical practice guidelines (38), and educational packages that involved the participation of opinion leaders (48) were evaluated in one RCT (38) and in one cluster RCT (48). Individual study results did not show a consistent and statistically significant effect on clinical management as a result of the KT intervention.

Evidence from two cluster RCTs $(43,44)$ showed that, compared with passive dissemination of clinical practice guidelines, KT interactive education interventions directed to health providers had a positive effect on patients' function at short and medium term. Statistically significant effects resulting from education-mediated $\mathrm{KT}$ interventions to health providers were not reported for other patient-related outcomes (ie, pain complaints, changes in work duty rate). There were inconsistent results regarding the effects of education-mediated KT interventions on other health provider outcomes such as knowledge about pain and pain management (reported as not statistically significant in one RCT [39] and one CBA [54] but statistically significant in one CCT [51]) and prescription behaviour (statistically significant in one cluster RCT [45] while not statistically significant in another cluster RCT [46]). One RCT (40) that compared the provision of written empirical evidence with a no-intervention group found a statistically significant improvement in health providers' confidence on therapeutic options derived from the KT intervention.

One CBA study (53) evaluated the effect of a professional intervention targeting physiotherapists that involved structural changes in the organization and delivery of care (ie, quality improvement) for low back pain. The study did not report statistically significant changes in clinical management, as evidenced by the number of clinical reports in which key assessment and management factors were documented.

KT interventions targeting patients: Three RCTs $(37,41,42)$ evaluated the effect of information/education and consumer decision aids (ie, written materials, oral and Internet-based education) offered to patients with low back pain $(37,41)$ and migraine (42) compared with usual care. Statistically significant results were reported for short-term changes in patients' function (37) and medium- and long-term changes in knowledge acquisition (41). Alternatively, no statistically significant differences were found between a patient-oriented KT education intervention and a nointervention group in the number of migraine-related complaints (42).

One RCT (22) evaluated a multifaceted patient-oriented KT intervention consisting of a coordination of care approach. Compared with usual care, the KT intervention did not have a statistically significant effect on the medium-term return-to-work rate.

KT interventions targeting combined groups: Two studies evaluated multifaceted KT interventions that targeted composite groups of health providers, patients and the community. One CCT (50) evaluated an interactive $\mathrm{KT}$ educational intervention whereas one CBA (52) assessed a mass media campaign. Both studies reported statistically significant effects on the medium-term appropriateness of prescription behaviours among health providers (50) and long-term beliefs about back pain in the community (52).

\section{DISCUSSION}

The present systematic review summarized the evidence from 19 studies on the effectiveness of a variety of KT interventions for chronic noncancer pain management. The review has revealed that professional KT interventions incorporating interactive education components have a positive effect on patients' function at the short- and medium-term. Evidence of the effectiveness of KT education interventions for health providers is variable for outcomes such as knowledge acquisition, prescription behaviour, clinical management and confidence in therapeutic options, and for other patient-related outcomes such as pain complaints and changes in work duty rates.

There is evidence that education, information and consumer decision aids directed at chronic noncancer pain patients can lead to improvements in functional capacity and knowledge about chronic pain issues. Evidence from one study on professional-oriented KT interventions on chronic noncancer pain issues that involve structural changes and quality improvement processes did not show significant effects on health provider- or patient-related outcomes. Similarly, there is currently little research evidence to support the effectiveness of other KT interventions that involve coordination of care approaches for chronic noncancer pain patients. More studies assessing the effects of KT interventions targeting structural changes and organization of care are needed to address these knowledge gaps.

The results of chronic pain treatment are particularly sensitive to the degree to which patients are prepared to accept the need for often-difficult changes in lifestyle and outlook. Knowledge is an essential component of behavioural change; however, the mere use of evidence-based knowledge about what to do when faced with chronic pain, even if performed effectively, may not result in measurable clinical improvements, simply because behavioural changes are often difficult to make. This is a limitation not of KT, but of the limited role that knowledge plays in influencing the complex nature of health-related behaviours.

The present systematic review included studies that used a variety of research designs (ie, cluster RCTs, RCTs, CCTs and CBAs). Consistent with the results of similar reviews (55), the majority of included studies were considered likely to be at high risk of bias. Although the majority of the research evidence on the effectiveness of KT interventions for chronic noncancer pain management was derived from RCTs or cluster RCTs, the risk of bias assessment was particularly challenging in many instances, due to incomplete or ambiguous reporting of methodological characteristics. A careful consideration and adoption of methods to reduce bias and enhance the transparency of reporting of primary trial research are necessary to expand the evidence base of KT interventions for the management of chronic noncancer pain.

A relatively heterogeneous group of KT interventions was evaluated in the studies, varying widely in their individual components and the level of action to support research implementation into practice. Overall, the poor reporting of details about their implementation (ie, theoretical framework of behaviour change, frequency and duration of the delivered intervention, characteristics and training of intervention providers, and implementation fidelity) across the studies has important consequences for the interpretation of the effectiveness outcomes in the present review. The lack of information from individual studies on how some procedures and other elements of the KT interventions were implemented as planned precluded the evaluation of whether certain KT interventions were ineffective due to an ineffectual intervention strategy or because they were poorly implemented $(56,57)$. For the KT interventions that showed promising results (ie, education approaches for health care providers and patients), the identification of the likely mechanism of action was equally hampered by poor reporting of KT implementation details. An improved reporting of these elements will facilitate a further understanding about what makes certain KT interventions effective, particularly those with multifaceted components, and the applicability and relevance of individual study findings to other situations (58). 
TABLE 3

Characteristics of intervention groups and knowledge translation (KT) effectiveness outcomes according to target population evaluated in individual studies

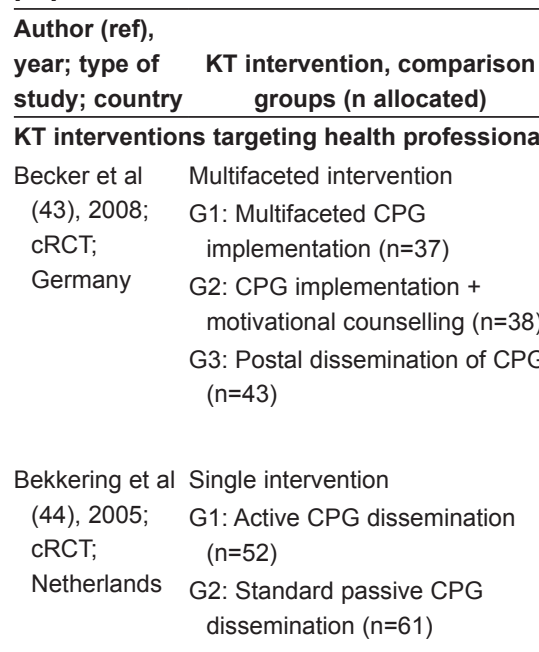

Derebery et al Single intervention

(49), 2002; G1: Active evidence-based

CCT; USA educational package $(n=61)$

G2: Usual pasive training $(n=151)$

Figueiras et al Single intervention

(45), 2001; G1: One-to-one education ( $n=98)$

cRCT; Spain G2: By-group education ( $n=98)$

Geraud et al Single intervention

(38), 2009; G1: E-learning education based on

$\mathrm{RCT}$; France $\mathrm{CPG}(\mathrm{n}=\mathrm{NR})$

G2: Live interactive workshop $(n=N R)$

Harris et al Single intervention

(39), 2008; G1: Online CME pain program

RCT; USA $\quad(n=49)$

G2: Live lectures $(n=50)$

G3: Control lecture $(n=55)$

Jones et al

(54), 1992;

Multifaceted intervention

CBA; USA

G1: Interactive educational program $(n=88)$

G2: Usual care $(n=87)$

Keijsers et al

(40), 1992;

RCT;

Single intervention

G1: Provision of written empirical evidence $(n=103)$

Netherlands G2: No intervention $(n=100)$

\begin{tabular}{ccc}
$\begin{array}{c}\text { Unit of } \\
\text { allocation, target, } \\
\text { clinical topic }\end{array}$ & Unit of analysis, numbers analyzed per group, primary outcome \\
and results & $\begin{array}{c}\text { Statistically } \\
\text { significant } \\
\text { result }\end{array}$ \\
\hline
\end{tabular}

Practice; GPs; Unit of analysis: Patient Yes

chronic LBP Function (Hannover Functional Ability Questionnaire for Measuring Back

Pain-related Functional Limitations [12 items]) (mean, 95\% Cl)

TOA $\quad \mathrm{G} 1(\mathrm{n}=\mathrm{NR}) \quad \mathrm{G} 2(\mathrm{n}=\mathrm{NR}) \quad \mathrm{G} 3(\mathrm{n}=\mathrm{NR})$

Baseline $\quad 67.5(21.4) \quad 68.7(20.9) \quad 65.8(21.9)$

6 months 72.9 (70.6 to 75.2$) 73.9(71.6 \text { to } 76.2)^{*} 70.2(68.8$ to 72.7$)$

12 months 72.9 (70.4 to 75.4$) 74.6$ (72.2 to 77.1$) 71.5$ (68.9 to 74.1$)$

${ }^{*} \mathrm{P}$ between groups $=0.032$

Practice; PTs; Unit of analysis: Patient

Yes

chronic LBP Physical functioning (Quebec Back Pain Disability Scale [(0 to 100 max]) (median score [IQR])

$\begin{array}{llll}\text { TOA } & \mathrm{G} 1(\mathrm{n}=48) & \mathrm{G} 2(\mathrm{n}=59) & \mathrm{MD}(95 \% \mathrm{Cl}) \\ \text { Baseline } & 38(26.5,50.5) & 40.5(26.3,55.8) & \\ 6 \text { weeks } & 24(13,40) & 17(4.6,32) & 1.9(-1.4 \text { to }-5.3)^{*} \\ 12 \text { weeks } & 20(7,32.8) & 17(4.6,32) & 2.8(-0.6 \text { to } 6.3) \\ 26 \text { weeks } & 20(7,32.8) & 11(4,29) & 4(0.6 \text { to } 7.3) \\ 52 \text { weeks } & 17(4.6,32) & 11(4,29) & 3.5(-0.2 \text { to } 7.3)\end{array}$

${ }^{*} \mathrm{P}=0.05$; overall effect $=4.88(\mathrm{P}>0.05)$

Health provider; Unit of analysis: Patient

OPs; LBP Patients' restricted work duty (rate)

Unable to

TOA $\quad$ G1 $(n=N R) \quad$ G2 $(n=N R)$

1 year $\quad 66.3 \quad 85$

$\mathrm{P}$ between groups $=\mathrm{NR}$

Geographical Unit of analysis: Health provider

Yes

area; GPs, FPs; Average prescribing behaviour improvement (prescription records review) (\%)

chronic pain TOA G1 $(n=94) \quad$ G2 $(n=59)$

9 months $\quad 6.5^{*} \quad 2.4$

$\mathrm{P}$ between groups $<0.05^{*}$

Health provider; Unit of analysis: Health provider

No

GPs; migraine Percentage of GP changing from a low or medium recommendation assimilation score (\%; recommendation assimilation score [1 to 10])

TOA $\quad \mathrm{G} 1(\mathrm{n}=198) \quad \mathrm{G} 2(\mathrm{n}=283)$

1 month $\quad 38.5$

$P$ between groups for equivalence test $>0.0001$

Health provider; Unit of analysis: Health provider

No

GPs, FPs; non- Physicians' knowledge about pain management (KnowPain-50 score)

malignant (mean $\pm \mathrm{SD}$ )

chronic pain

$\begin{array}{llll}\text { TOA } & \mathrm{G} 1(\mathrm{n}=30) & \mathrm{G} 2(\mathrm{n}=32) & \mathrm{G} 3(\mathrm{n}=33) \\ \text { Baseline } & 143.6 \pm 19.7 & 138.0 \pm 17.5 & 139.2 \pm 18.7 \\ 3 \text { months } & 149.5 \pm 21.4 & 151.0 \pm 19.4 & 144.8 \pm 22.0\end{array}$

$P$ between groups $=0.448$

Practice; nurses; Unit of analysis: Health provider

chronic pain Nurses' knowledge about pain (36 knowledge items [true/false]) (\% mean score)

TOA $\quad$ G1 $(n=74) \quad$ G2 $(n=75)$

Baseline $69 \quad 68$

$\begin{array}{lll}\text { NR } & 71 & 67\end{array}$

$P$ between groups: NR

Health provider; Unit of analysis: Health provider

GPs, PTs; Reduction in confidence in back schools (VAS scale [5\% to $95 \%$; maximum:

chronic LBP very effective]) (baseline to end point mean change, \%)

$\begin{array}{lll}\text { TOA } \quad \mathrm{G} 1(\mathrm{n}=82) & \mathrm{G} 2(\mathrm{n}=88) \\ 10 \text { weeks } \quad-10 \%{ }^{*} & 1.6 \% \\ { }^{*} \mathrm{P} \text { between groups }<0.05 & \end{array}$ 
TABLE 3 - CONTINUED

Characteristics of intervention groups and knowledge translation (KT) effectiveness outcomes according to target population evaluated in individual studies

\begin{tabular}{lc}
$\begin{array}{l}\text { Author (ref), } \\
\text { year; type of }\end{array}$ & KT intervention, comparison \\
study; country & groups ( allocated) \\
\hline $\begin{array}{l}\text { KT interventions targeting health professionals - } \\
\text { Rahme et al }\end{array}$ & Multifaceted intervention \\
(46), 2005; & G1: Workshop and decision tree \\
CRCT; & (n=84) \\
Canada & G2: Workshop alone $(n=29)$ \\
& G3: Decision tree alone $(n=54)$ \\
& G4: No intervention ( $n=82)$ \\
Smelt et al & Single intervention \\
(47), 2010; & G1: Proactive education intervention \\
CRCT; & ( $n=N R)$ \\
Netherlands & G2: Usual care ( $=N R)$ \\
&
\end{tabular}

Stevenson et Single intervention al (48), 2006; G1: Educational package $(n=17)$ cRCT; United G2: Usual in-service training $(n=13)$ Kingdom

Ferguson et al Single intervention

(53), 2010; G1: Quality improvement and audit

CBA; $(n=N R)$

Scotland G2: Pre-intervention period $(n=N R)$

KT interventions targeting chronic noncancer pain patients Coudeyre et al Single intervention
(37), 2006;
G1: The Back Book $(n=72)$
RCT; France
G2: Usual care $(n=70)$ LBP

$\begin{array}{ccc}\text { Unit of } & & \text { Statistically } \\ \begin{array}{c}\text { allocation, target, } \\ \text { clinical topic }\end{array} & \text { Unit of analysis, numbers analyzed per group, primary outcome } & \text { significant } \\ \text { and results } & \text { result }\end{array}$

Town; GPs; Unit of analysis: Prescriptions

No

osteoarthritis Number of adequate prescriptions (prescription score) (\%)

$\begin{array}{lllll}\text { TOA } & \text { G1 } & \text { G2 } & \text { G3 } & \text { G4 } \\ \text { Baseline } & 58 & 51 & 51 & 47 \\ 5 \text { months } & 62 & 56 & 54 & 49\end{array}$

G1 versus G4 OR ( $95 \% \mathrm{Cl}): 1.9$ (0.9 to 3.8$)$; G2 versus G4 OR (95\% Cl): 5.7

(0.4 to 26.9$)$; $\mathrm{G} 3$ versus $\mathrm{G} 4 \mathrm{OR}(95 \% \mathrm{Cl}): 1.0$ (0.6 to 1.7$)$

Health provider; Unit of analysis: Patient

No

Headache complaints (Headache Impact Test) (mean change score between groups)
TOA
6 months 0.05 points
$\mathrm{G} 2(\mathrm{n}=\mathrm{NR})$
$P$ between groups $=0.083$

Health provider; Unit of analysis: Health provider

OPs; LBP Percentage correct answers in knowledge test (45 true/false questions) (mean $\pm S D$ )

$\begin{array}{llll}\text { TOA } & \mathrm{G} 1(\mathrm{n}=18) & \mathrm{G} 2(\mathrm{n}=20) & \mathrm{MD}(95 \% \mathrm{Cl}) \\ \text { Baseline } & 70 \pm 11.7 & 67 \pm 10.4 & \\ \text { NR } & 85 \pm 6.8 & 73 \pm 6.7 & 9(95 \% \mathrm{Cl} 0.4 \\ & & & \text { to } 16)^{*}\end{array}$

*P between groups $<0.05$

Practice; PTs; Unit of analysis: Health provider

Change in clinical management

Time spent providing advice about work situation (discharge summary questionnaire) (\%)

$\begin{array}{llll}\text { TOA } & \text { G1 }(n=16) & \text { G2 }(n=11) & \text { OR }(95 \% \text { Cl }) \\ 6 \text { months } & 37 \% & 35 \% & 1.1(0.05 \text { to } 2.5)\end{array}$

Practice; PTs; Unit of analysis: Process measure

chronic LBP Case notes with LBP factor documented (\%) (medical record audit)

Unable to

TOA $\quad \mathrm{G} 1(\mathrm{n}=\mathrm{NR}) \quad \mathrm{G} 2(\mathrm{n}=\mathrm{NR})$

7 months 79

$\mathrm{P}$ between groups $=\mathrm{NR}$

Patient; chronic

Unit of analysis: Patient

Functional capacity (Quebec Back Pain Disability Scale [0 to 100 max]) (mean \pm SD)

$\begin{array}{lcc}\text { TOA } & \text { G1 }(n=63) & \text { G2 }(n=62) \\ \text { Baseline } & 48.4 \pm 14.5 & 52.1 \pm 16.8 \\ 3 \text { months } & 34.5 \pm 18.4 & 42.4 \pm 14.9 \\ \text { *P between groups }=0.03 & \end{array}$

Patient; chronic Unit of analysis: Patient

LBP Patients' LBP knowledge acquisition (mean effect size; rating scale [0 to 45])

TOA G1 $(n=181) \quad$ G2 $(n=163)$

6 months eta ${ }^{2}=0.056^{*}$

12 months eta ${ }^{2}=0.026^{*}$

${ }^{*} \mathrm{P}$ between groups $<0.001$

Patient; migraine Unit of analysis: Clinical encounter

Number of migraine-related topics discussed during the visit (single score from survey) (mean number)

$\begin{array}{lll}\text { TOA } & \text { G1 }(n=28) & \text { G2 }(n=22) \\ \text { NR } & 5.5 & 4.3\end{array}$

$P$ between groups $=\mathrm{NR}$ 
TABLE 3 - CONTINUED

Characteristics of intervention groups and knowledge translation (KT) effectiveness outcomes according to target population evaluated in individual studies

\begin{tabular}{|c|c|c|c|c|}
\hline $\begin{array}{l}\text { Author (ref), } \\
\text { year; type of } \\
\text { study; country }\end{array}$ & $\begin{array}{l}\text { KT intervention, comparison } \\
\text { groups ( } \mathrm{n} \text { allocated) }\end{array}$ & $\begin{array}{c}\text { Unit of } \\
\text { allocation, target, } \\
\text { clinical topic }\end{array}$ & $\begin{array}{l}\text { Unit of analysis, numbers analyzed per group, primary outcome } \\
\text { and results }\end{array}$ & $\begin{array}{c}\text { Statistically } \\
\text { significant } \\
\text { result }\end{array}$ \\
\hline
\end{tabular}

KT interventions targeting chronic noncancer pain patients - Continued

Rossignol et al Multifaceted intervention

Patient; chronic Unit of analysis: Patient

No

\begin{tabular}{lllll} 
(22), 2000; & G1: Coordination of primary care & LBP & \multicolumn{3}{l}{ Return to work (medical record audit) $(\%)$} \\
RCT;Canada & program $(n=54)$ & TOA & G1 $(n=48)$ & $G 2(n=48)$ \\
& G2: Usual care $(n=56)$ & 6 months & 77.8 & 73.2
\end{tabular}

\section{Combined target groups}

Doubova et al Multifaceted intervention

(50), 2010; G1: Interactive educational

CCT; Mexico programme (patients, $n=282$; health providers, $n=58$ )

G2: Passive educational programme (patients, $n=291$; health providers, $\mathrm{n}=58$ )

Buchbinder

et al (52), G1: Victorian Work Cover Authority

2005; CBA; back campaign (patients, $n=1185$;

Australia health providers, $n=691$ )

G2: No intervention (patients, $n=1185$; health providers $n=691$ )
Health provider; Unit of analysis: Health provider

Yes

patients, FPs; $\quad$ Proportion of appropriately prescribed NOAs (medical record audit) $(\%$ [95\% CI])

nonmalignant TOA G1 $(n=58) \quad$ G2 $(n=52)$

pain syndrome Baseline 65.2 (59.5 to 71.0) 62 (56.1 to 67.9 );

6 months $\quad 82.6$ (78 to 87.2$) \quad 68$ (62.8 to 73.2$)$

MD between groups: $15.0(95 \% \mathrm{Cl} 14.4 \text { to } 16.3)^{*}$

Geographical Unit of analysis: Patient

area; GPs; back Patients' beliefs about back pain (Back Beliefs Questionnaire [9 to 45, more pain positive]) (mean score, $95 \% \mathrm{Cl}$ )
TOA
$\mathrm{G} 1(\mathrm{n}=900)$
$\mathrm{G} 2(\mathrm{n}=600)$
$\operatorname{MD}(95 \% \mathrm{Cl})$
Baseline
26.5 (26.1 to 26.8 ) 26.3 (25.9 to 26.6$) \quad 0.2(0.3 \text { to }-0.8)^{*}$
Survey 2
28.4 (27.9 to 28.8 ) 26.2 ( 25.7 to 26.7 ) 2.1 (1.4 to 2.8$)^{*}$
Survey 3
29.7 (29.2 to 30.3 ) 26.3 (25.7 to 26.8 ) 3.5 (2.7 to 4.2$)^{*}$
3 years
28.8 (28.4 to 29.2 ) 26.1 (25.5 to 26.6 ) 2.7 (2.1 to 3.4$)^{*}$
*P between groups $<0.05$

*Statistically significant difference between groups. CBA Controlled before-and-after study; CCT Controlled clinical trial; CME Continuing medical education; CPG Clinical practice guideline; cRCT Cluster randomized controlled trial; G Group; GP General physician; FP Family physician; IQR Interquartile range; LBP Low-back pain; MD Mean difference; NOA Nonopioid analgesic; NR Not reported; OP Occupational physician; PT Physical therapist; RCT Randomized controlled trial; ref Reference; TOA Time of outcome assessment; VAS Visual analogue scale

The adoption of theoretical frameworks for KT, such as the Promoting Action on Research Implementation Health Services (PARIHS) model (59-61), may help to expand our understanding of the role of KT interventions in managing chronic noncancer pain. The PARIHS framework recognizes the complexities and nonlinearity of implementing evidence into practice. The unique aspect of this framework is that it recognizes the complexity of interactions between professionals and the clinical context, and emphasizes the role of process of implementation by facilitation as an important factor. This model has resonance with the 'Knowledge to Action' model introduced by Graham et al (62) which highlights a range of contextual factors that need to be taken into consideration when implementing evidence.

Assessing the impact of a $\mathrm{KT}$ intervention requires the consideration of how research is used and how it is expected to impact clinical practice (63). More than one-half of the studies in the present review used measures of change in health provider outcomes to assess the effectiveness of $\mathrm{KT}$ interventions. Measures of change in patient outcomes were assessed in nine studies and included both measures of actual change in patients' health status (eg, patient function) and surrogate measures of patients' change in health status (eg, knowledge acquisition and beliefs about pain, work duty rate and return to work). The pattern of reporting outcome evaluation measures in health practitioner and patients in the individual studies is similar to that identified in similar reviews $(55,64)$. The complexity of chronic noncancer pain suggests that multiple domains are relevant when evaluating the effects of $\mathrm{KT}$ interventions. It is recommended that authors of future studies agree on key domains of outcomes to enable the harmonization of measuring the benefits of KT interventions for the management of chronic noncancer pain across studies. Similarly, it is important that future studies refine measures of the multidimensional aspect of patient-related outcomes to include role vocational, social and emotional aspects as outcome measures, and include the patient perspective on what represents a clinically meaningful change that may be expected as a result of KT interventions.

Overall, the KT interventions for chronic noncancer pain management identified in the present systematic review involved both instrumental and conceptual uses of knowledge for the implementation of research into practice (65). Instrumental use of research included the implementation of clinical practice guidelines and facilitating process of change and quality monitoring. Conceptual uses of research through the $\mathrm{KT}$ interventions in the present review supported the acquisition of new knowledge about chronic noncancer pain issues and increased awareness of knowledge gaps through the dissemination of research evidence.

Some results of the present study are consistent with those of previous systematic reviews of $\mathrm{KT}$ in the area of cancer pain management $(55,66)$ in which comprehensive educational interventions (eg, information, behavioural instructions and advice in relation to pain management by means of verbal, written, audio- or videotaped or computer-aided modalities) were significantly more likely to have positive results compared with those that did not use this approach. In their review, Cummings et al (55) found that education interventions addressed to health providers were associated with significant decreases in patients' pain intensity, and increases in health providers' knowledge and attitudes about cancer pain. Similarly, they found that more than one-half of the studies involving educational KT interventions targeting patients identified a significant decrease in patients' pain intensity as a result of the intervention.

Bennett et al (66) performed a systematic review and meta-analysis to evaluate the benefit of patient-based educational interventions (eg, information, behavioural instructions and advice) in relation to management of cancer pain, which are given by a health provider or peer. 
They found that educational interventions improved knowledge and attitudes among cancer pain patients.

Results of our review are also consistent with overviews of systematic reviews of dissemination and implementation strategies of research findings $(58,67,68)$, which have found a consistent effect across interventions including a combination of educational outreach visits, reminders, audit and feedback, social marketing and interactive educational meetings.

The strengths of the present systematic review pertain to its rigour in searching the literature, the criteria-based selection of relevant evidence, the rigorous appraisal of study validity and the evidence-based inferences. Although the identification of a small number of studies reporting comparable outcome measures precluded visual assessment of potential publication bias using funnel plots, the comprehensive search strategy is likely to have identified most of the available literature on the effectiveness of $\mathrm{KT}$ interventions for chronic noncancer pain management. Another important asset of the present systematic review is the control for the impact of multiple-publication bias in the narrative synthesis of the results. Multiple publications from single studies can bias a review in a number of ways. Because studies with significant results are more likely to lead to multiple publications and presentations (69) they are more likely to be located in literature searches and, if not recognized as such, they can lead to an overestimation of the intervention effects in systematic reviews $(70,71)$. Our review identified nine multiple publications, avoiding the inclusion of duplicate publications of data, which may have skewed the evidence base in the analysis of the study results.

The present systematic review has several limitations that must be noted. A variety of KT intervention taxonomies are available in the scientific literature and there is a lack of agreement within the research community on a common theoretical or empirical framework for classifying KT interventions. We used a standard taxonomy of interventions aimed at achieving practice change that has been widely used in similar reviews $(55,72-74)$. We recognize that many discrete KT interventions deployed various elements that could be classified in more than one category (eg, educational interventions plus patient reminders, audit and feedback techniques, or local opinion leaders).

Planned KT strategies are more likely to be successful if the choice of the approach is informed by an assessment of the likely

\section{REFERENCES}

1. Henry JL. The need for knowledge translation in chronic pain. Pain Res Manag 2008;13:465-76.

2. Cote P, Kristman V, Vidmar M, et al. The prevalence and incidence of work absenteeism involving neck pain: A cohort of Ontario losttime claimants. Spine 2008;33:S192-8.

3. Leroux I, Dionne CE, Bourbonnais R, Brisson C. Prevalence of musculoskeletal pain and associated factors in the Quebec working population. Int Arch Occup Environ Health 2005;78:379-86.

4. Juurlink DN, Herrmann N, Szalai JP, Kopp A, Redelmeier DA. Medical illness and the risk of suicide in the elderly. Arch Intern Med 2004;164:1179-84.

5. Becker N, Thomsen AB, Olsen AK, Sjogren P, Bech P, Eriksen J. Pain epidemiology and health related quality of life in chronic nonmalignant pain patients referred to a Danish multidisciplinary pain center. Pain 1997;73:393-400.

6. Schopflocher D, Taenzer P, Jovey R. The prevalence of chronic pain in Canada. Pain Res Manag 2011;16:445-50.

7. Moulin DE, Clark AJ, Speechley M, Morley-Forster PK. Chronic pain in Canada - prevalence, treatment, impact and the role of opioid analgesia. Pain Res Manag 2002;7:179-84.

8. Toth C, Lander I, Wiebe S. The prevalence and impact of chronic pain with neuropathic pain symptoms in the general population. Pain Med 2009; 10:918-29.

9. Guerriere DN, Choiniere M, Dion D, et al. The Canadian STOP, PAIN project - Part 2: What is the cost of pain for patients on waitlists of multidisciplinary pain treatment facilities? Can J Anaesth 2010;57:549-58. barriers and facilitators that can operate at the patient and health care provider level and beyond (eg, structural, organizational, peergroup barriers and clinical encounter barriers). The present systematic review was limited to the analysis of the effectiveness of KT interventions for chronic noncancer pain management and did not evaluate the role of different levels of barriers and facilitators to KT. An examination of these factors is crucial to understand how KT interventions can best be implemented. Such analysis implies going beyond the experimental evidence to incorporate qualitative approaches.

\section{CONCLUSIONS}

Professional KT interventions in the area of chronic noncancer pain that incorporate interactive education lead to positive effects on patients' function in the short- and medium-term. The benefits of these interventions on other outcomes are not consistent. Patientbased educational interventions can result in benefits in function and knowledge about pain. There is little research evidence to support the effectiveness of KT interventions that involve structural changes and quality improvement processes or coordination of care approaches for chronic noncancer pain patients. Future studies in the area of KT for chronic noncancer pain management should provide better details of the interventions implementation and use consistent theoretical frameworks to inform appropriate choices for dissemination and implementation strategies and to improve their estimate of the effectiveness of such interventions.

ACKNOWLEDGEMENTS: The authors thank Dr Jessica Moffatt and Mr Kenneth Bond for their assistance with titles and abstracts screening, and Dr Carmen Moga for helping with study selection and risk of bias assessments.

DISCLOSURES: The authors have no competing interests to declare.

FUNDING: This research received funding from the Community Alliances for Health Research \& Knowledge Exchange on Pain, a Canadian research network funded by the Canadian Institutes of Health Research (Grant number: FRN 86786).

10. Phillips CJ, Schopflocher D. The Economics of chronic pain. In: Rashiq S, Schopflocher D, Taenzer P, Jonsson E, eds. Chronic Pain: A Health Policy Perspective. Weinham: Wiley-Blackwell, 2008:41-50.

11. Ramage-Morin P, Gilmour H. Chronic pain at ages 12 to 44: Health matters. Ottawa: Statistics Canada, 2010. Statistics Canada Catalogue No. 82-003-XPE. <www.statcan.gc.ca/pub/82-003x/2010004/article/11389-eng.pdf $>$ (Accessed July 15, 2012).

12. Millar WJ. Chronic pain. Ottawa: Statistics Canada, 1996. Catalogue No. 82-003. <www.statcan.gc.ca/pub/82-003-x/1995004/ article/2819-eng.pdf $>$ (Accessed July 15, 2012).

13. Mäntyselkä $P$, Kumpusalo E, Ahonen R, et al. Pain as a reason to visit the doctor: A study in Finnish primary health care. Pain 2001;89:175-80.

14. Blyth FM, March LM, Nicholas MK, Cousins MJ. Chronic pain, work performance and litigation. Pain 2003;103:41-7.

15. Sheehan J, McKay J, Ryan M, Walsh N, O'Keeffe D. What cost chronic pain? Irish Med J 1996;89:218-9.

16. Piwko C, Desjardins OB, Bereza BG, et al. Pain due to multiple sclerosis: Analysis of the prevalence and economic burden in Canada. Pain Res Manag 2007;12:259-65.

17. Ekman M, Jonhagen S, Hunsche E, Jonsson L. Burden of illness of chronic low back pain in Sweden: A cross-sectional, retrospective study in primary care setting. Spine 2005;30:1777-85.

18. Stewart WF, Ricci JA, Chee E, Morganstein D. Lost productive work time costs from health conditions in the United States: Results from the American Productivity Audit. J Occup Environ Med 2003;45:1234-46. 
19. Stewart WF, Ricci JA, Chee E, Hirsch AG, Brandenburg NA. Lost productive time and costs due to diabetes and diabetic neuropathic pain in the US workforce. J Occup Environ Med 2007;49:672-9.

20. Van Leeuwen MT, Blyth FM, March LM, Nicholas MK, Cousins MJ. Chronic pain and reduced work effectiveness: The hidden cost to Australian employers. Eur J Pain 2006;10:161-6.

21. Chou R. Evidence-based medicine and the challenge of low back pain: Where are we now? Pain Practice 2005;5:153-78.

22. Rossignol M, Abenhaim L, Seguin P, et al. Coordination of primary health care for back pain: A randomized controlled trial... including commentary by Cherkin D. Spine 2000;25:251-9.

23. McGuirk B. Safety, efficacy, and cost effectiveness of evidencebased guidelines for the management of acute low back pain in primary care. Spine 2001;26:2615-22.

24. Hagen EM, Grasdal A, Eriksen HR. Does early intervention with a light mobilization program reduce long-term sick leave for low back pain: A 3-year follow-up study. Spine 2003;28:2309-15.

25. Canadian Institutes of Health Research. About knowledge translation. 2009. <www.cihr-irsc.gc.ca/e/29418.html> (Accessed March 19, 2013).

26. Bostrom AM, Slaughter SE, Chojecki D, Estabrooks CA. What do we know about knowledge translation in the care of older adults? A scoping review. J Am Med Dir Assoc 2012;13:210-9.

27. Woolf SH. The meaning of translational research and why it matters. JAMA 2008;299:211-3.

28. Eccles MP, Mittman BS. Welcome to implementation science. Implement Sci 2006;1:1.

29. Grol R, Grimshaw J. From best evidence to best practice: Effective implementation of change in patients' care. Lancet 2003;362:1225-30

30. Straus S, Tetroe J, Graham ID. Knowledge Translation in Healthcare: Moving from Evidence to Practice. West Sussex: Wiley-Blackwell/BMJ, 2008.

31. Cochrane Effective Practice and Organisation of Care Review Group. Data collection checklist. < http://epoc.cochrane.org/sites/ epoc.cochrane.org/files/uploads/datacollectionchecklist.pdf> (Accessed February 6, 2011).

32. Higgins JP, Altman DG, Sterne JA. Assessing risk of bias in included studies. In: Higgins JPT, Green S, eds. Handbook for Systematic Reviews of Interventions Version 5.1.0 (updated March 2011). The Cochrane Collaboration, 2011. <www.cochranehandbook.org > (Accessed October 10, 2011).

33. Henry JL. The need for knowledge translation in chronic pain. Pain Res Manag 2008;13:465-76.

34. Popay J, Roberts H, Sowden A, et al. Guidance on the conduct of narrative synthesis in systematic reviews. <www.lancs.ac.uk/shm/ research/nssr/index.htm> (Accessed June 7, 2012).

35. Glujovsky D, Bardach A, Garcia Marti S, Comande D, Ciapponi A. New software for early stage of systematic reviews. XVIII Cochrane Colloquium. The Joint Colloquium of the Cochrane \& Campbell Collaborations. Keystone, October 18 to 22, 2010.

36. Ciapponi A, Glojovsky D, Bardach A, Garcia Marti S. EROS: A new software for early stage of systematic reviews. HTAi 2011 Conference. Rio de Janeiro, June 27 to 29, 2011.

37. Coudeyre E, Givron P, Vanbiervliet W, et al. The role of an information booklet or oral information about back pain in reducing disability and fear-avoidance beliefs among patients with subacute and chronic low back pain: A randomized controlled trial in a rehabilitation unit. Ann Readapt Med Phys 2006;49:600-8.

38. Geraud G, Valade D, Meric G, Troy S, Lanteri-Minet M. Study of the impact of a general practitioner educational program in managing migraine according to the French guidelines (FAST study). 4th Congress of the International Headache Society. Philadelphia, September 10 to 13, 2009:50-1.

39. Harris JM Jr, Elliott TE, Davis BE, Chabal C, Fulginiti JV, Fine PG. Educating generalist physicians about chronic pain: Live experts and online education can provide durable benefits. Pain Med 2008;9:555-63.

40. Keijsers JF, Bouter LM, Meertens RM, Kessels AG, Knipschild PG. The impact of back school research on the beliefs of health care professionals: A randomised survey of general practitioners and physiotherapists. Physiother Theory Pract 1992;8:79-83.

41. Meng K, Seekatz B, Roband H, Worringen U, Vogel H, Faller H. Intermediate and long-term effects of a standardized back school for inpatient orthopedic rehabilitation on illness knowledge and self-management behaviors: A randomized controlled trial. Clin J Pain 2011;27:248-57.

42. Sciamanna CN, Nicholson RA, Lofland JH, Manocchia M, Mui S, Hartman CW. Effects of a website designed to improve the management of migraines. Headache 2006;46:92-100.

43. Becker A, Leonhardt C, Kochen MM, et al. Effects of two guideline implementation strategies on patient outcomes in primary care: A cluster randomized controlled trial. Spine 2008;33:473-80.

44. Bekkering GE, van Tulder MW, Hendriks EJ, et al. Implementation of clinical guidelines on physical therapy for patients with low back pain: Randomized trial comparing patient outcomes after a standard and active implementation strategy. Phys Ther 2005;85:544-55.

45. Figueiras A, Sastre I, Tato F, et al. One-to-one versus group sessions to improve prescription in primary care: A pragmatic randomized controlled trial. Med Care 2001;39:158-67.

46. Rahme E, Choquette D, Beaulieu M, et al. Impact of a general practitioner educational intervention on osteoarthritis treatment in an elderly population. Am J Med 2005;118:1262-70.

47. Smelt A, Blom J, Dekker F, et al. A proactive approach towards migraine patients in general practice: A pragmatic randomized controlled trial. 2nd European Headache and Migraine Trust International Congress - EHMTIC. Nice, October 28 to 31, 2010:S27.

48. Stevenson K, Lewis M, Hay E. Does physiotherapy management of low back pain change as a result of an evidence-based educational programme? J Eval Clin Pract 2006;12:365-75.

49. Derebery VJ, Giang GM, Saracino G, Fogarty WT. Evaluation of the impact of a low back pain educational intervention on physicians' practice patterns and patients' outcomes. J Occup Environ Med 2002;44:977-84.

50. Doubova SV, Mino-Leon D, Reyes-Morales H, Flores-Hernandez S, Torres-Arreola LP, Perez-Cuevas R. Effects of two educational programmes aimed at improving the utilization of non-opioid analgesics in family medicine clinics in Mexico. J Eval Clin Pract 2010;16:716-23.

51. Smits PB, Verbeek JH, Van Dijk FJ, Metz JC, ten Cate TJ. Evaluation of a postgraduate educational programme for occupational physicians on work rehabilitation guidelines for patients with low back pain. Occup Environ Med 2000;57:645-6.

52. Buchbinder R, Jolley D. Effects of a media campaign on back beliefs is sustained 3 years after its cessation. Spine 2005;30:1323-30.

53. Ferguson F, Holdsworth L, Rafferty D. A national framework for supporting improvements in the physiotherapy assessment and management of low back pain: The Scottish experience. Physiotherapy 2010;96:198-205.

54. Jones KR, Fink R, Pepper G, et al. Improving nursing home staff knowledge and attitudes about pain. Gerontologist 2004;44:469-78.

55. Cummings GC, Armijo-Olivo S, Biondo PD, et al. Effectiveness of knowledge translation interventions to improve cancer pain management. J Pain Symptom Manag 2011;41:915-39.

56. Higgins JP, Deeks JJ. Selecting studies and collecting data. In: Higgins JPT, Green S, eds. Handbook for Systematic Reviews of Interventions Version 5.1.0 (updated March 2011). The Cochrane Collaboration. < cochrane-handbook.org > (Accessed October 12, 2011).

57. Dusenbury L, Brannigan R, Falco M, Hansen WB. A review of research on fidelity of implementation: Implications for drug abuse prevention in school settings. Health Educ Res 2003;18:237-56.

58. Grimshaw JM, Shirran L, Thomas R, et al. Changing provider behavior: An overview of systematic reviews of interventions. Med Care 2001;39:II-2-25.

59. Kitson AL, Rycroft-Malone J, Harvey G, McCormak B, Seers K, Titchen A. Evaluating the successful implementation of evidence into practice using the PARIHS framework: Theoretical and practical challenges. Implement Sci 2008;3. doi:10.1186/1748-5908-3-1.

60. Kitson AL, Harvey G, McCormack B. Enabling the implementation of evidence based practice: A conceptual framework. Qual Health Care 1998;7:149-58.

61. Rycroft-Malone J, Kitson AL, Harvey G, et al. Ingredients for change: Revisiting a conceptual framework. Qual Saf Health Care 2002;11:174-80.

62. Graham IM, Stewart M, Hertog MG, Cardiovascular Round Table Task Force. Factors impeding the implementation of cardiovascular prevention guidelines: Findings from a survey conducted by the European Society of Cardiology. Eur J Cardiovasc Prev Rehabil 2006;13:839-45. 
63. Conner RF. Measuring evaluation utilization: A critique for different techniques. In: Ciarlo JA, ed. Utilizing Evaluation: Concepts and Measurement Techniques. Beverly Hills: Sage, 1981:59-75.

64. Hakkennes S, Green S. Measures for assessing practice change in medical practitioners. Implement Sci 2006;1:29.

65. Beyer JM. Research utilization: Bridging the gap between communities. J Manage Inquiry 1997;6:17-22.

66. Bennett MI, Bagnall AM, Jose Closs S. How effective are patientbased educational interventions in the management of cancer pain? A systematic review and meta-analysis. Pain 2009;143:192-9.

67. Bero LA, Grilli R, Grimshaw JM, Harvey E, Oxman AD, Thomson MA. Closing the gap between research and practice: An overview of systematic reviews of interventions to promote the implementation of research findings. BMJ 1998;317:465-8.

68. Boaz A, Baeza J, Fraser A, European Implementation Score Collaborative Group (EIS). Effective implementation of research into practice: An overview of systematic reviews of the health literature. BMC Research Notes 2011;4:212.
69. Easterbrook PJ, Berlin JA, Gopalan R, Matthews DR.

Publication bias in clinical research. Lancet 1991;337:867-72.

70. Tramer MR, Reynolds DJ, Moore RA, McQuay HJ. Impact of covert duplicate publication on meta-analysis; a case study. BMJ 1997;315:635-40.

71. Huston P, Moher D. Redundancy, disaggregation, and the integrity of medical research. Lancet 1996;347:1024-6.

72. Thompson DS, Estabrooks CA, Scott-Findlay S, Moore K, Wallin L. Interventions aimed at increasing research use in nursing: A systematic review. Implement Sci 2007;2:15.

73. Scott S, Albretch L, O'Leary K, et al. Systematic review of knowledge translation strategies in the allied health professions. Implement Sci 2012;7:70.

74. Grimshaw JM, Thomas RE, Maclennan G, et al. Effectiveness and efficiency of guideline dissemination and implementation strategies. Health Technol Assess 2004;8:iii-iv, 1-72. 


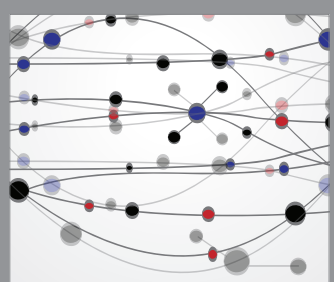

The Scientific World Journal
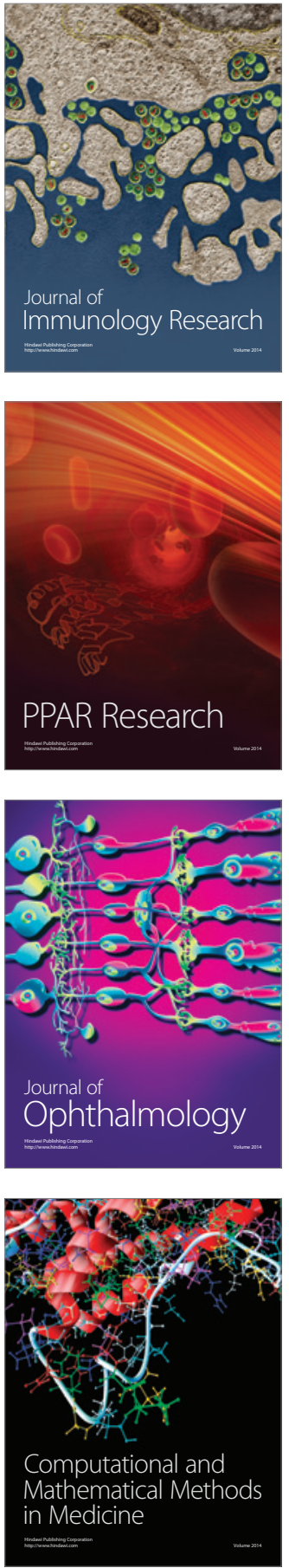

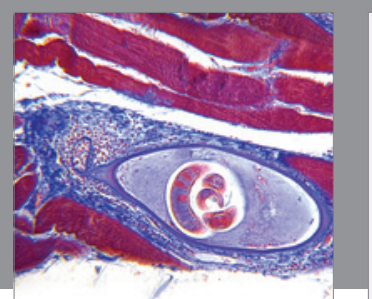

Gastroenterology Research and Practice

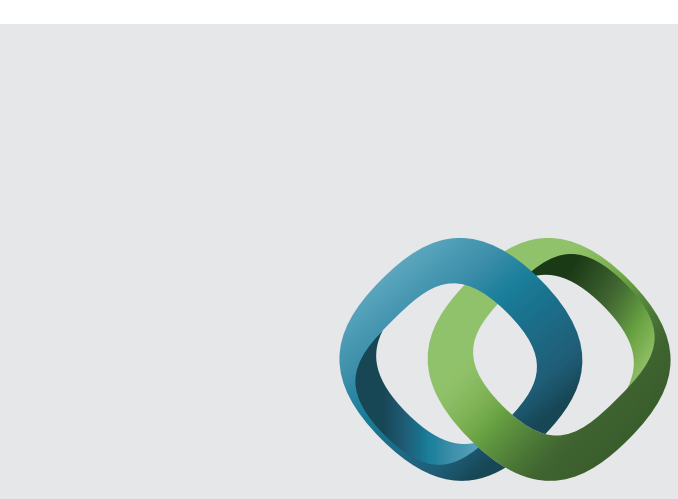

\section{Hindawi}

Submit your manuscripts at

http://www.hindawi.com
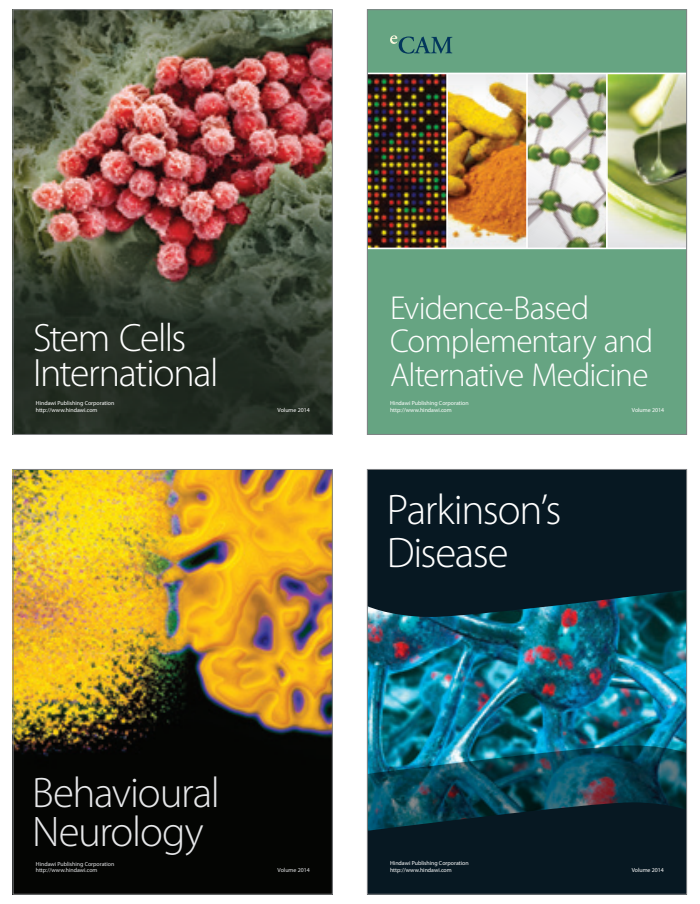
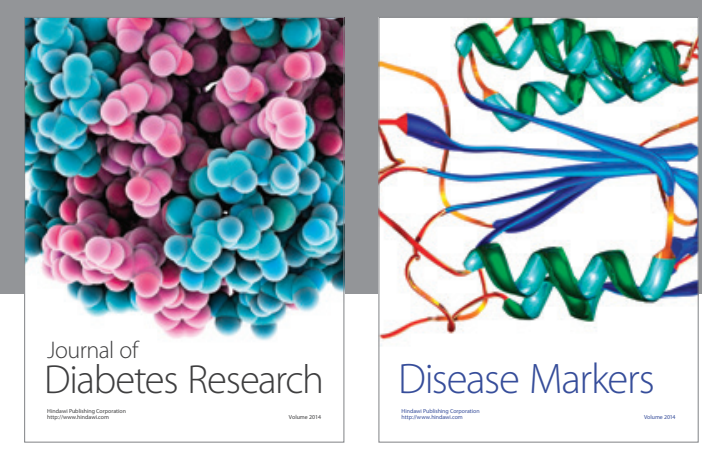

Disease Markers
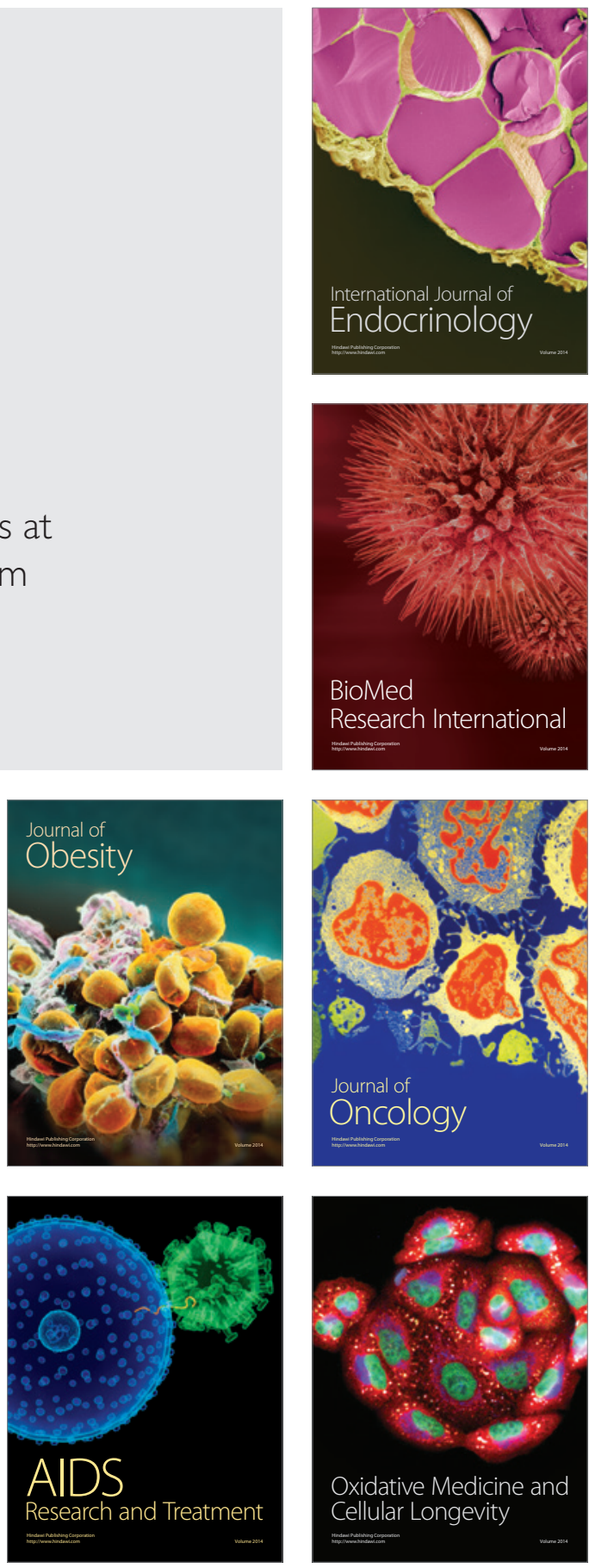